\title{
Avionics Frequency Response Characteristics Testing System
}

\author{
Feng Tian*, Fei Sun and Xinyu Chen \\ College of Automation, Shenyang Aerospace University, Shenyang, China \\ feixiang006@yeah.net
}

\begin{abstract}
To meet the demand of avionics control system's frequency response characteristic and the testing, a new avionics frequency response testing system is proposed. The design uses PXI series card of NI Corporation and the software programming is used in the design, to realize the function of the testing signal's generation, the collection of the response stgnal, the processing of the sampling data and the mapping of the system's frequency response characteristic curve, and so on. In this paper, the hardware composition, softwareldesign, testing steps, and the processing method of data sampling are mainly introduced. The results of application show that the proposed system is economical and eliable. It has a good stability and can meet the avionics system frequercy response testing requirements. It not only achieves all the collection of the response signal, analysis and calculation, but also achieves the data storage and playback. The proposed system vas the advantages of simple and quick, flexible configuration, portable low cost, and so on.
\end{abstract}

Keywords: Frequency Response Characteristic; Testing System; Data Processing

\section{Introduction}

With the development of electronic technology and aerospace applications, avionics system has become an important part of the aircraft. The system's frequency response characteristic is the key technology of the avionics system. Because it not only has an important influence on the whole control system's reliability and stability, but also has important implications for circuit analysis and troubleshooting. Frequency response characteristic paameters can intuitively reflect the stability of the system, and provide a data support to improve the control characteristic of this system. In order to obtain the avionics system frequency lesponse characteristic parameters in time, an avionics frequency response testing system is designed. The hardware design mainly adopts PXI series chip of NI Corporation, at the same time, using self-made hardware circuit to filter the sampling data The software design adopts VC to write the testing system's each module, algorithm and so on. It can realize the function of the testing signal's generation, the collection of the response signal, the processing of the sampling data and the mapping of the system's frequency response characteristic curve, so as to meet the frequency response characteristics of the testing requirements.

\section{The Composition and Working Principle of the System}

HP35670A is the traditional dynamic signal analyzer which can take 15 minutes for each test. The instrument is expensive; complicated operation and relatively simple configuration. Considering the signal selection, sampling precision, test time, low cost and portable, this system mainly includes the following parts: Signal generation and acquisition system, display system, PC software and so on. The overall design of the system is shown in Figure 1. 


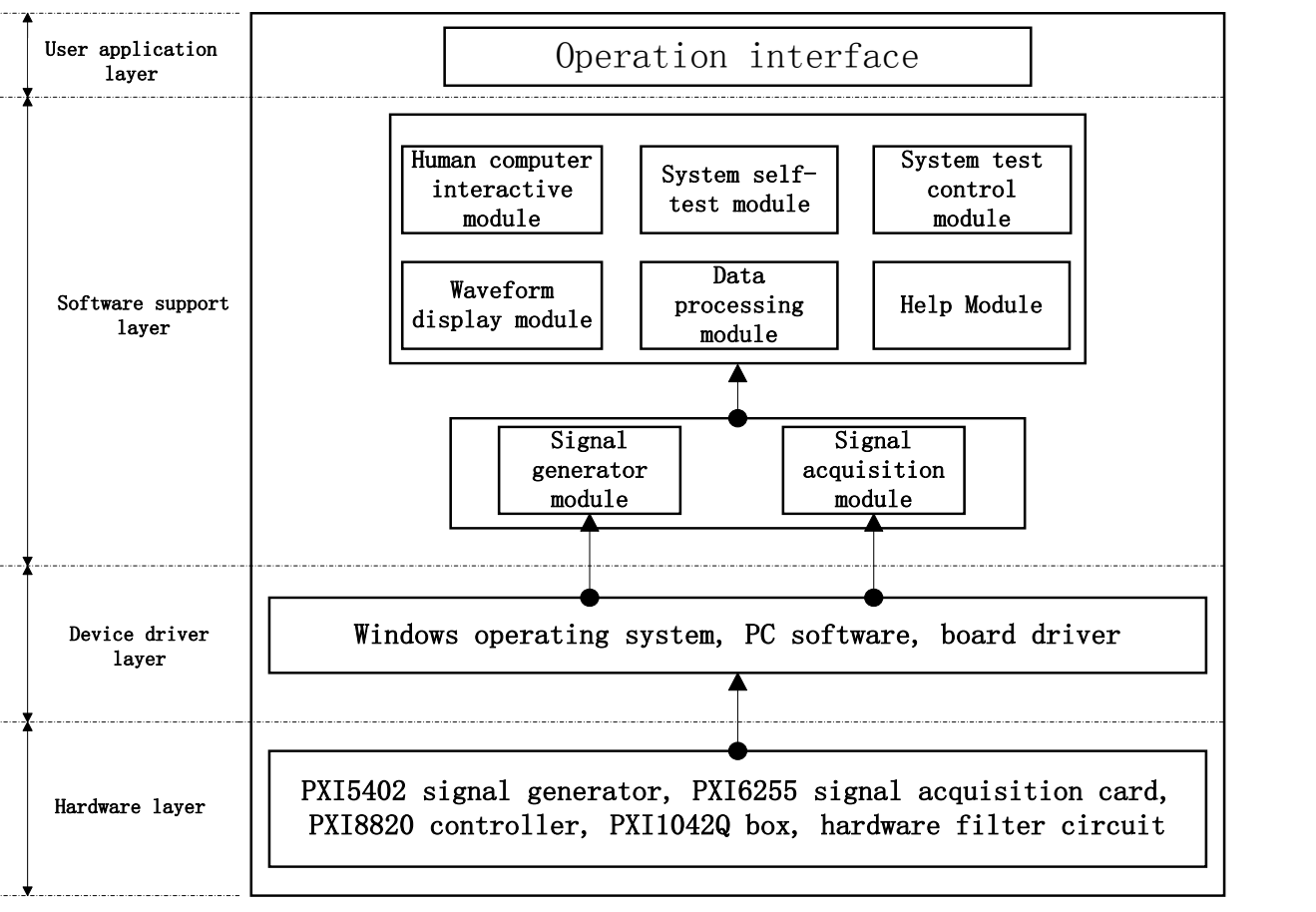

Figure 1. The Overall Design Diagram of the System

The overall design of the system mainl includes hardware layer, device driver layer, software support layer and user application layer. The hardware is based on the platform if the NI PXI-1042 chassis. The chassis 1s installed on the controller card which is as the CPU of the whole system, and with the signal generator and the DAQ board which includes the multifunction data acquisition. Those cards can be directly installed in the slot of the chassis of the PXI, which is convenent and simple. The device driver layer provides the operating platform for software design, the software design mainly uses $\mathrm{VC}++$. Including the sweep signal generator module, response signal acquisition module, data processing module, human-computer interaction module, system self-test and help module.

The working principle of the system is shown in Figure 2.

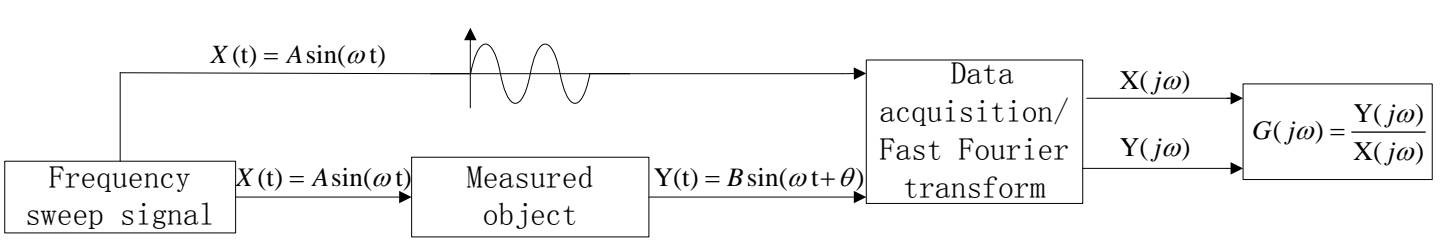

\section{Figure 2. The Working Principle of the System}

The-sine signal is loaded into the object being measured, at the same time, the response signal and the frequency sweep signal of the system are collected. After the data processing, the two kinds of signal's amplitude and phase comparison are compared. Then measured gain and phase difference of the system under test are obtained, and the Bode diagram can be drawn, and the transfer function can be calculated.

\section{The Realization of the System Hardware and Software Design}

According to the basic principle of this system. Mainly uses the signal generator PXI5402, signal collecting card PXI-6255, box PXI-1042, controller PXI-8820 of National Instruments. The hardware principle and object diagram are shown in Figure 3. 

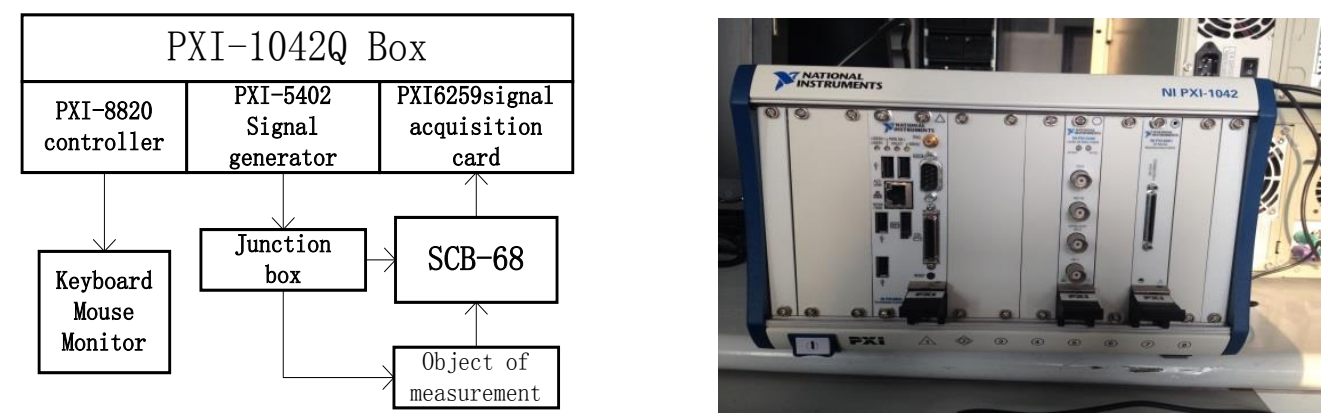

Figure 3. The Hardware Principle and Object Diagram

\subsection{PXI Series Card}

PXI-5402 is a signal generator which can generate the sweep signal of this system. Built-in sine, square, triangle, noise and DC offset functions, 400MS/s effective sample rate with interpolation; $0.355 \mu \mathrm{Hz}$ frequency resolution. It can be controlled by VC programming. PXI-6255 is high-speed multifunction data acquisition card to collect the input and output data. The voltage range of the data acquisition is $\pm 10 \mathrm{~V}$, Maximum update rate is $1.25 \mathrm{MS} / \mathrm{s}$, This device have NI-MCal calibration technology for improved measurement accuracy and six DMA channes for high-speed data throughput. The NI PXI-8820 high-value embedded controller features an Intêl dual-core Celeron 1020E processor and is designed for PXI and CompactPC, systems. With $2.2 \mathrm{GHz}$ base frequency and $1333 \mathrm{MHz}$ DDR3L standard memory. It can be equipped with Win7 system, Visual Studio2010 programming software and so on. The NI PXI-1042/PXI1042Q 8-slot chassis are designed to meet the needs of a wide range of test and measurement applications. The PXI-1042 operates in a temperature range extended to $55^{\circ} \mathrm{C}$. The PXI-1042 offers quieter operation, with acoustic emissions as low as $43 \mathrm{db}$.

\subsection{Band Pass Filtêr}

The frequencr range of the avionics system is $0.1 \mathrm{~Hz} \sim 100 \mathrm{~Hz}$, the range belongs to the low frequency, due to the fact that the field test environment is complex, the superposition of all kinds of noise, it has very large interference on the system, and it need to add a band pass filter in the signal-channel, remove high-frequency unwanted signals, as far as possible let the low frequency signal through the channel without attenuation. In order to minimize effect of filter corner frequency, the cut-off frequency of the filter is generally greater than 3 times more than that of the effective signal frequency, however, the sampling frequency should be 2 3 times the cutoff frequency of the filter.

The working principle of band pass filter is shown in Figure 4. 


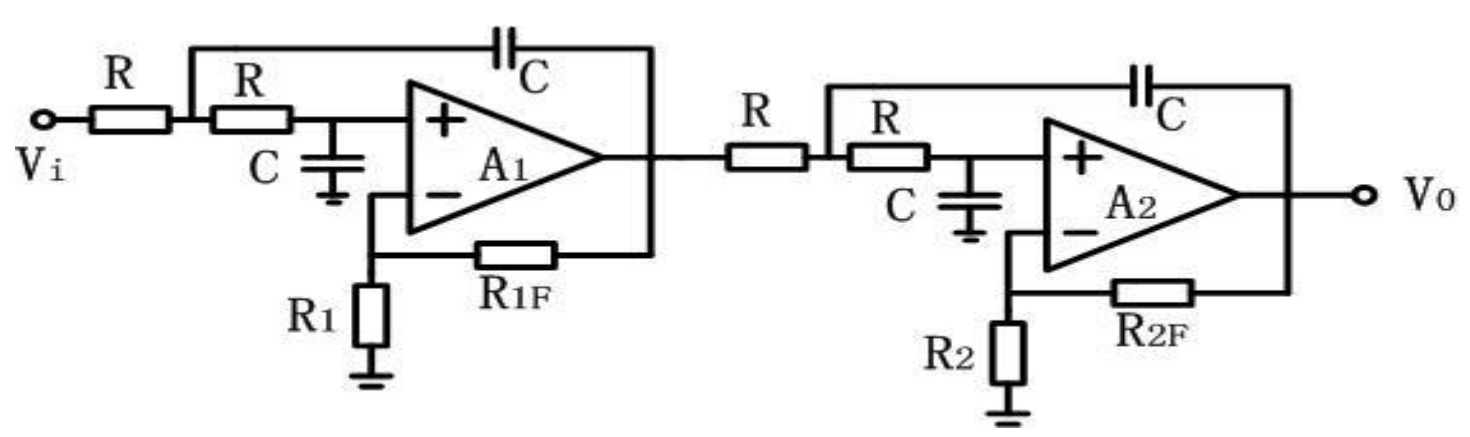

Figure 4. Band Pass Filter

The cutoff frequency of the filter used in this system is $1 \mathrm{KHz}$, According to the design requirements of the various device parameters $R_{1}=R_{2}=10 K \Omega \quad C=0.1 \mu F \quad R=1.6 K \Omega$

\section{The Design of System Software}

The system software mainly use Visual Studio 2010 programning software, specifically refers to the use of the Visual $\mathrm{C}++$ system, including the man-machine interaction module, help module, system self-test module, system/control module, interface test waveform display module, response data processing module and so on. The testing system software overall uses hierarchical design, each layer services the upper, and meanwhile they as the lower customer, in this way, we can allow a complex problem into a sequence of incremental steps. The test software is divided into hardware layer, driver layer, and support layer and application layer software in accordance with the hierarchical structure. The modular relationship of software architecture as shown in Figure 1. The device driver may froyide access to the hardware equipment for the upper layer software; the software support layet contains the various equipment control with API function, the various functional modules realize algorithm, dynamic link library design and so on. The user application layer is to provide users with a friendly manmachine interface which is simple and convenient, so that it can implement system testing, process monitoring and other functions under the various functions control algorithm support.

\subsection{The System Self-test Control Module and Board Control Module}

The system self-test podule work is to complete their own state before the examination for the system, testing signal generator for the system and availability of multifunctional data acquisition card, and completing each card initialization, calibration etc. The system hardware self-test process is shown in Figure 5. 


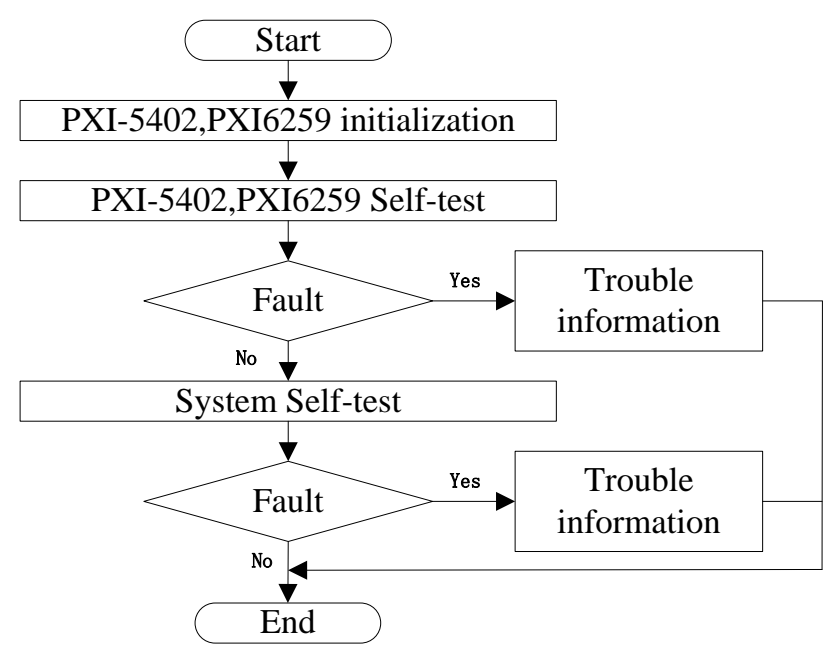

Figure 5. The Self-Test Of System

The board control module is used to control PXI-6255 and PXI5402 mainly, Using the underlying API function hardware products, you can call Visual Studio 2010 development environment, and complete control of the hardware board. This module/mainly refers to the PXI5402 signal generator to produce different frequency sweep signal, the PXI-6255 is used to collection frequency response signal for system, and the process as shown in Figure 6.

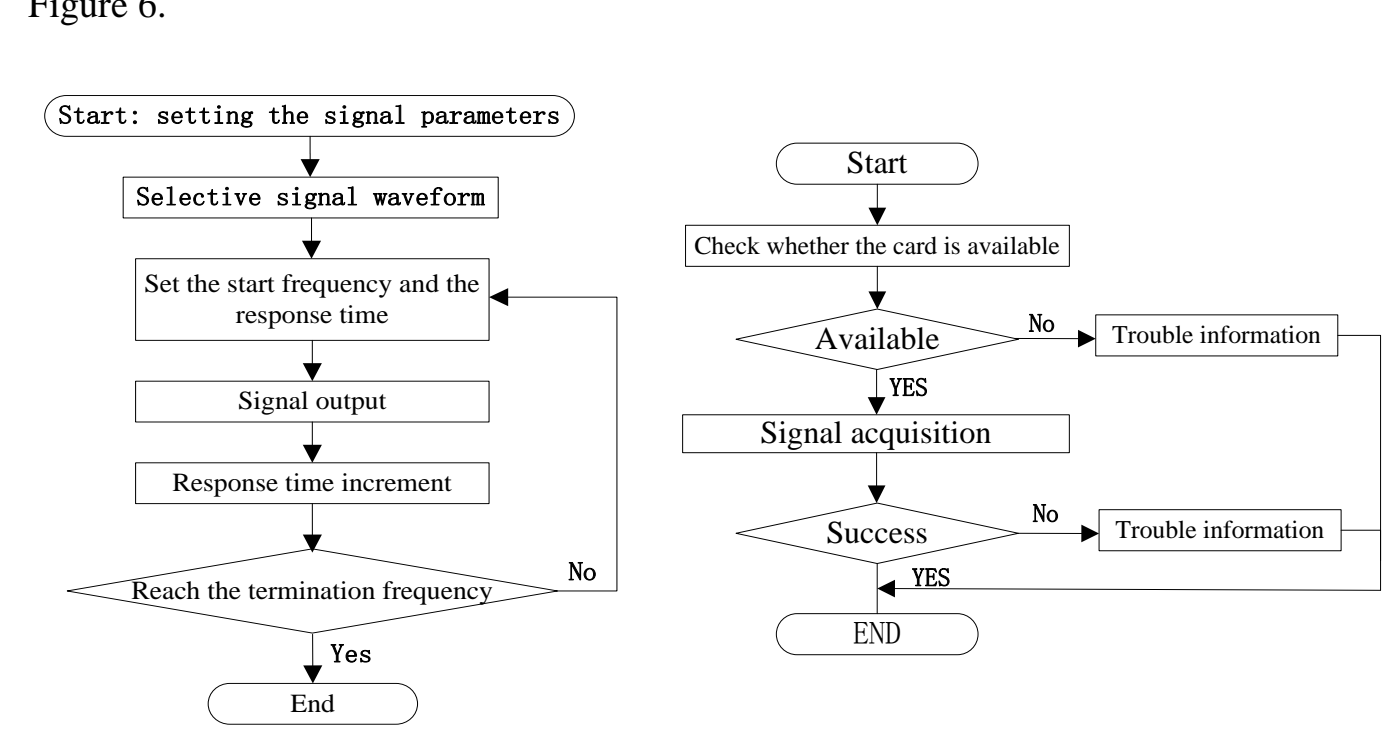

Figure 6. The Board Control Module

\section{Response Data Processing Module}

Response data processing module is mainly used to achieve the frequency response data processing, calculate the frequency response characteristics and draw the Bode diagram. Direct express frequency response characteristics, Provide data support for the improvement of system control performance. The method of data processing will be introduced in the next chapter. 


\subsubsection{Data Preprocessing}

In the process of avionics system control the aircraft rudder, vibration signal is produced. Then, we collect the vibration signal data. Because with the temperature changes the amplifier will produce zero drift, and outside the frequency range of the sensor low frequency performance is not stable, and there also have the environmental disturbance around the sensor. The data often deviates from the baseline and even deviates from the baseline will change by the time. The frequency response data preprocessing is to remove the trend and intercept steady data. The engineering practice will generally reject the first three periodic signals; the feedback signal for the remaining is steady signal. Algorithm of signal preprocessing as follows:

$$
Y_{m}(\mathrm{t})=Y(\mathrm{t})-\frac{\max Y(\mathrm{t})+\min Y(\mathrm{t})}{2}
$$

Among of them, $Y(\mathrm{t})$ is the original data, $Y_{m}(\mathrm{t})$ is preprocessed data.

Because the measured object is a nonlinear system, the external environment in the process of testing will make noise. Linear filtering method can deal with noise, but most of the linear filter with low pass characteristics. Removing the noise also-makes the waveform's details and edge blur. Median filter is a nonlinear method for rem oving noise It can not only achieve noise suppression, filter pulse jamming and graphic scanning noise, but also overcome the details of the image blurring caused by the linear filter, keep the edge information of the image. It can be good to remove the noise of the curve.

The filter window length is $n=2 k+\mathbb{N}$ the number of measurements is $M(M>>n)$ Observations: $u\left(t_{1}\right), u\left(t_{2}\right), \cdot, u\left(t_{M}\right)$.Median filter output:

$$
\operatorname{median}\left[u\left(t_{i}\right)\right]= \begin{cases}u\left(t_{k+1}\right), & n=2 k+1 \\ \frac{1}{2}\left[u\left(t_{k}\right)+u\left(t_{k+1}\right)\right], & n=2 k\end{cases}
$$

Among of them $u\left(t_{k}\right)$ express in the vindow $n=2 k+1$ observations of the $\mathrm{K}$ maximum or minimumnalue.

According to the above definition window $n=2 k+1$, the relationship of one dimensional median method iltering is $X\left(t_{i}\right)=$ median $\left[u\left(t_{i-k}\right) \cdot \mathcal{u}\left(t_{i}\right), \cdots, u\left(t_{i+k}\right)\right]$.The median filter window length is $n=2 k+1$, But when the yidth range of the pulse signal is smaller than the $k$, a median filtering method willeliminate the signal. So the properties of median filter can do processing details in noise removal.

4.2.2 Sin Signal: When sin signal is the sweep signal of the system, we use the FFT to calculate the amplitude and phase frequency characteristics. FFT is used to establish the relation hip between the time conversion function and spectrum function. "Time" or "frequency" selects continuous value or discrete value, which forms the variety forms of DFT. For a $\mathrm{N}$ sequence of discrete FFT formula

$$
X(\mathrm{k})=\sum_{n=1}^{N} X(\mathrm{n}) \times \mathrm{e}^{(-\mathrm{j} \times 2 \pi \times(\mathrm{k}-1) \times(\mathrm{n}-1) / \mathrm{N})} \quad 1 \leq k \leq N
$$

Because of the DTF is periodic and symmetric, so using frequency extraction of FFT can greatly improve the speed of operation, but this method requires zero filling operation on the sampling signal, characteristic in the nature of FFT shows that the spectrum zero does not change the signal's spectrum. Because the test time of each frequency point is integer times of the signal cycle, and in order to avoid the leakage of the spectrum, so in data processing usually uses the window like spectral analysis, which can get better results. 


\subsubsection{Chirp Signal}

System adopts Chirp signal as the frequency sweep signal, the frequency sweep signal and the response signal of the system are collected. Then, according to the principle of mutual power spectrum, the frequency response characteristics of the system under test are calculated. Chirp wave, also known as linear frequency modulation pulse waveform, its expression is:

$$
Y_{i}=A * \sin ((0.5 a * i+b) * i)
$$

Among of them, $i=0,1,2, \cdots, n-1$, A is amplitude, $a=2 \pi\left(f_{2}-f_{1}\right) / n, b=2 \pi f_{1}$ where $f_{1}$ as the starting frequency, $f_{2}$ as the end frequency, $n$ as the number of samples sample.

Using chirp continuous wave as a frequency sweep signal to have the test and crosspower spectrum is used to calculate its frequency response characteristics, which describes the amplitude and the phase relationship between input and output signals. Frequency response function is obtained by the cross power spectrum of the signa input, output and the auto power spectrum of input signal. The calculated data can get crosspower spectrum amplitude and phase data, and the amplitude data of the input signal's auto power spectrum. Then, the cross power spectrum and the auto power spectrum of the corresponding item division is the system frequency response amplitude sequence, and the cross power spectrum phase data is the phase change on the system frequency response. The above data can be used to draw the bird figure and calculate the transfer function.

\subsection{The Man-machine Interface and Help Module}

The human-computer interaction module is used to build a set of software interface for the user to use the various functions the software with completing the detection of legitimacy of user interaction information and the enabled state adjustment of interface elements. The software interface is showin ingure 7.

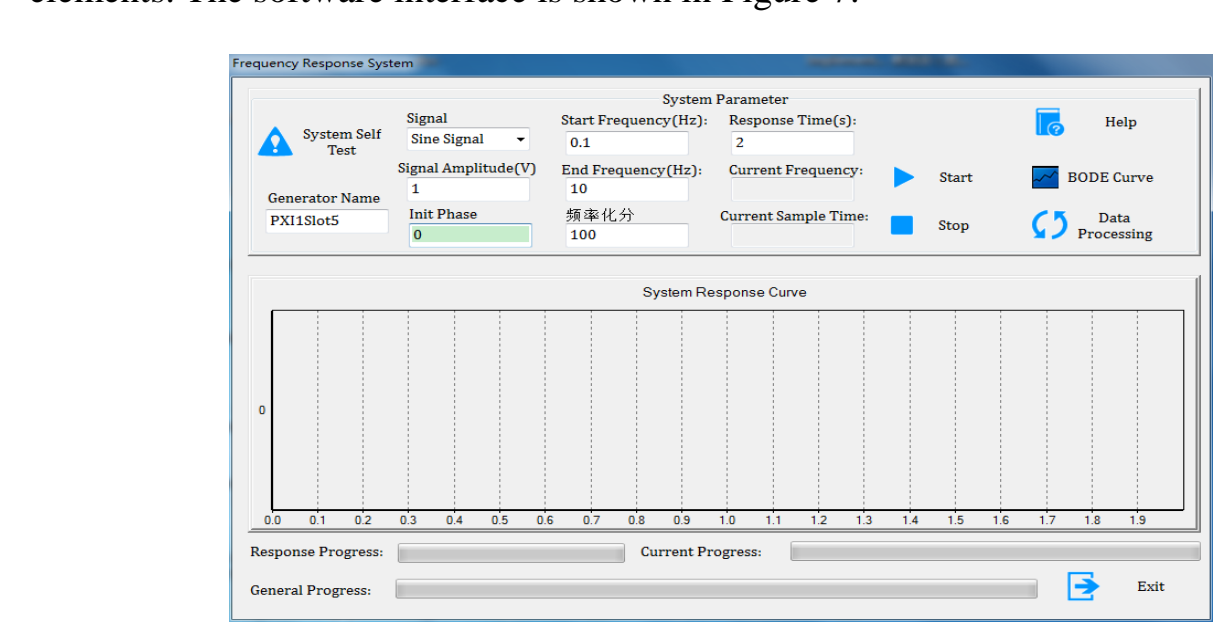

Figure 7. Human-Computer Interaction Interface

The interface includes the display of card name, the input module including the dropdown box selection of sweep signal, amplitude and phase of sweep signal, the initial frequency and stop frequency selection, data processing button, Bode diagram button, and the response curve of the system display.

The help module contains the complete description of the test system, tutorial and the solutions of common problems and abnormal problems and document content, helping software personnel master the various function of the whole test system, 
providing the guide of common troubleshooting. The interface contains the "help" button, and click it to get help document.

\section{Experimental Results}

In engineering practice, according to the actual situation, a certain aircraft avionics system frequency response has been tested. Using this system and the dynamic signal analyzer HP35670A to test the system at the same time, and make a data comparison. Calculating the amplitude gain and phase difference of each frequency point, and drawing the bode diagram. Using the two kinds of methods, the Bode diagrams are measured by the instruments as shown in Figure 8.
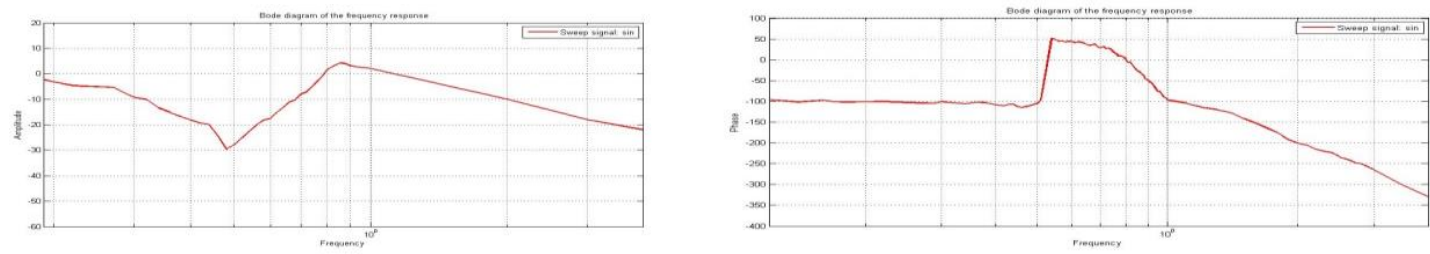

(a) Sweep signal is sin signal

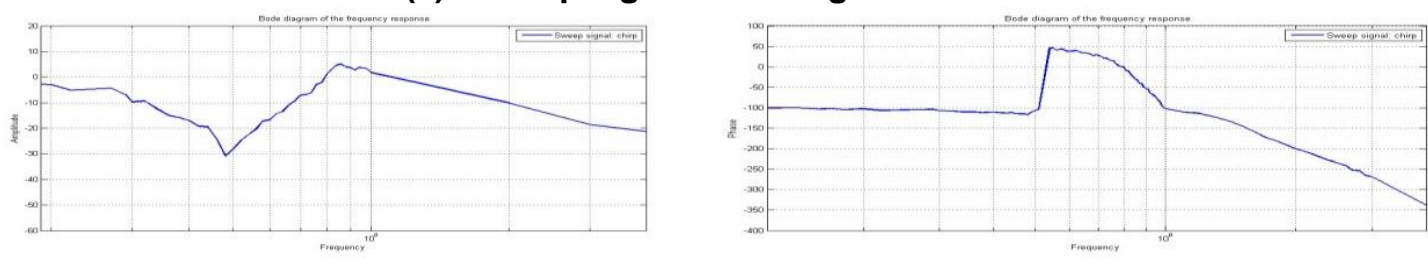

(b) Sweep signal is chirp signal

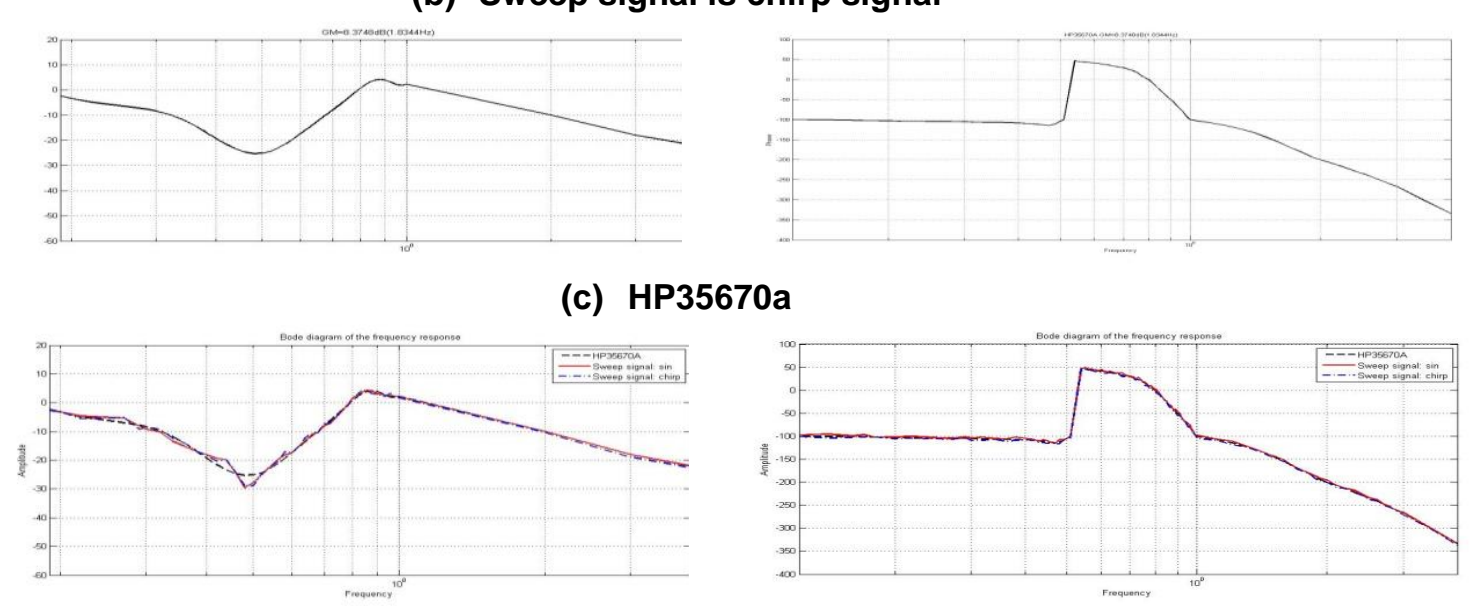

(d) Comparison

Figure 8. Bode Diagram

It can be seen clearly from the Figure 8 that diagram of this system's testing data coincide well with $35670 \mathrm{~A}$ data. It shows that the system can accurately measure the frequency of avionics system. If you need a further analysis of the system's transfer function, you can make use of the related functions in the matlab software, set relevant parameters to make the transfer function fitting directly.

Calculate the error between two methods of the testing system and dynamic signal analysis instrument. The accuracy of the testing system reaches $0.1 \%$. 


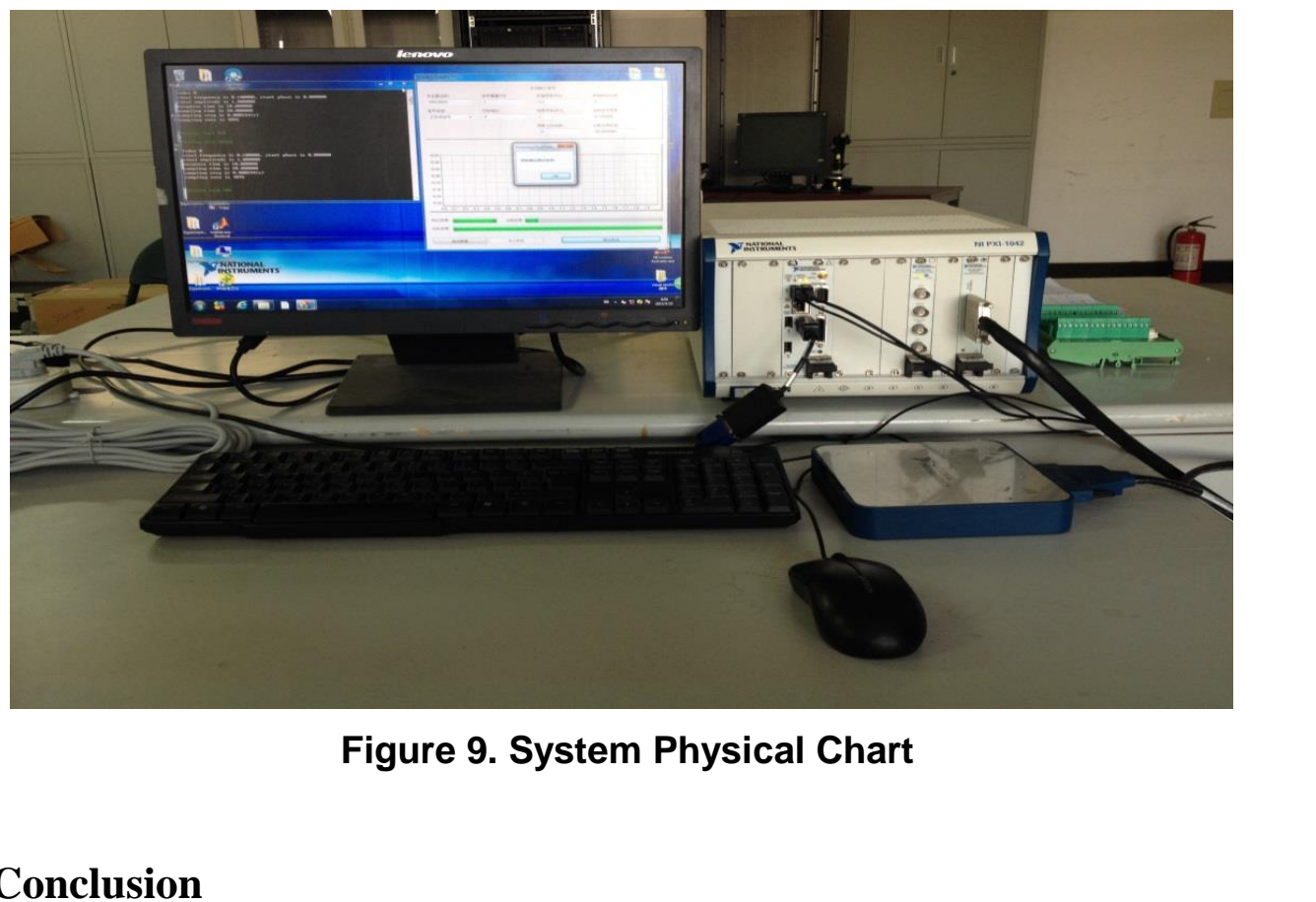

\section{Conclusion}

The design of avionics frequency testing system, which realized the function of the testing signal's generation, the collection of the response signal, the processing of the sampling data and the mapping of the system's frequency response characteristic curve, and so on System has the characteristics of friendly manmachine interaction, feature-rich and flexible extension, which make the system have high reliability and god stability. The system has the advantages of convenient connection, strong anti-interference and high testing precision by using PXI technology. Compare with the raditional dynamic signal analysis instrument, the system is reliable economical, good stability, high precision and can satisfy the requirement of avionics systemis frequency response testing.

\section{References}

[1] G. Wang, Q. Gu, M. Wang and L. Zhang, "Research on the architecture of the new generation integrated avionics system", J.Chinese Journal of Aeronautics, (2014.06), pp. 1473-1486.

[2] Q. Ma and K. Huang, "Research on frequency characteristic test system based on PC", J. Computer Measurement \& Control, (2005.10), pp. 10-13.

[3] Y. Guo, L.Z. Guo, S.A. Billings Z.Q. DanielCoca and A. Lang, "Parametric frequency response method for non-liner time-varying systems", J. International Journal of Systems Science, (2014), pp. 4510.

[4] M. e, "The angle vibration frequency response test system based on LabVIEW", J. Aviation Precision Manufaeturing Technology, (2011.04), pp. 60-62.

[5] Y. Shen, X.Cui, J. Ma and W. Niu, "Trusted software technology of integrated avionics system", J. Chinese Journal of Aeronautics, (2009.05), pp. 938-945.

[6] H. Zhang, T. Cui, X. Cheng and J. Yang, "Frequency response test system of mining equipment of multi-channel audio number", J. Electro acoustic technology, (2013.09), pp. 24-28+31.

[7] M. Gretinger, M. Secara, Cl. Festila and E. H Dulf, "'Chirp' signal generators for frequency response experiments", C. Proceedings of 2014 IEEE International Conference on Automation, Quality and Testing, Robotics, AQTR, (2014).

[8] D.F. Reyes-Romero, A.S. Cubukcu and G.A. Urban, "Measurement and simulation of the frequency response of a thermal flow sensor at different flow speeds", J. Sensors \& Actuators: A.Physical, vol. 189, (2013).

[9] F. Zhang, W. Chu, X. Fan and M. Wan, "Research on the structure of Integrated Modular Avionics System”, J. Electronics Optics \& Control. (2009.09), pp. 47-51+59.

[10] S. Peng, J. Xiao, Q. Ma and A. Wang, "Research on frequency characteristic test system based on dSPACE", J . Microelectronics and computer, (2008.11), pp. 222-224. 


\section{Authors}

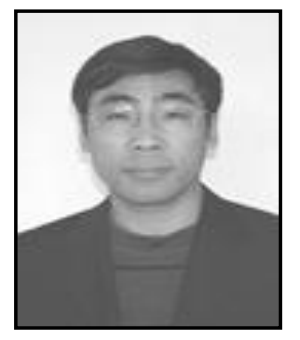

Feng Tian, he received the Ph.D. degree in detection technology and automatic equipment from Northeastern University, China, in 2004. He is currently professor at the College of Automation of Shenyang Aerospace University. He is a member of $\mathrm{CCF}$, and a doctoral tutor. His current research focuses on wireless sensor networks, internet of things, embedded systems and intelligent control.

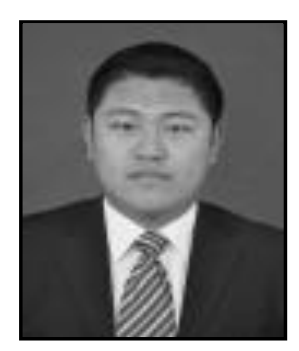

Fei Sun, he received the bachelor degree in electric Observation and control technology and instrument from Northeastern University, China, in 2012. He is currently studying for a Master degree in the control engineering of Shenyang Aerospace University.

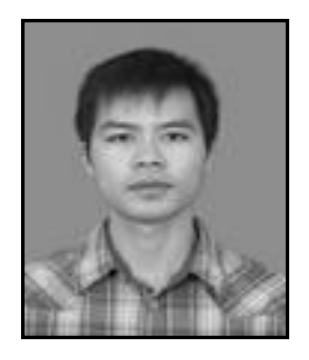

Xinyu Chen, he received the Ph.D. degree in control theory and control engineering from Dalian Maritime University, China, in 2014. He is currently lecturer at the College of Automation of Shenyang Aerospace Unversity. His eurrent research focuses on Machine vision, wireless senser networks, internet of things, embedded systerns and intelligent control.

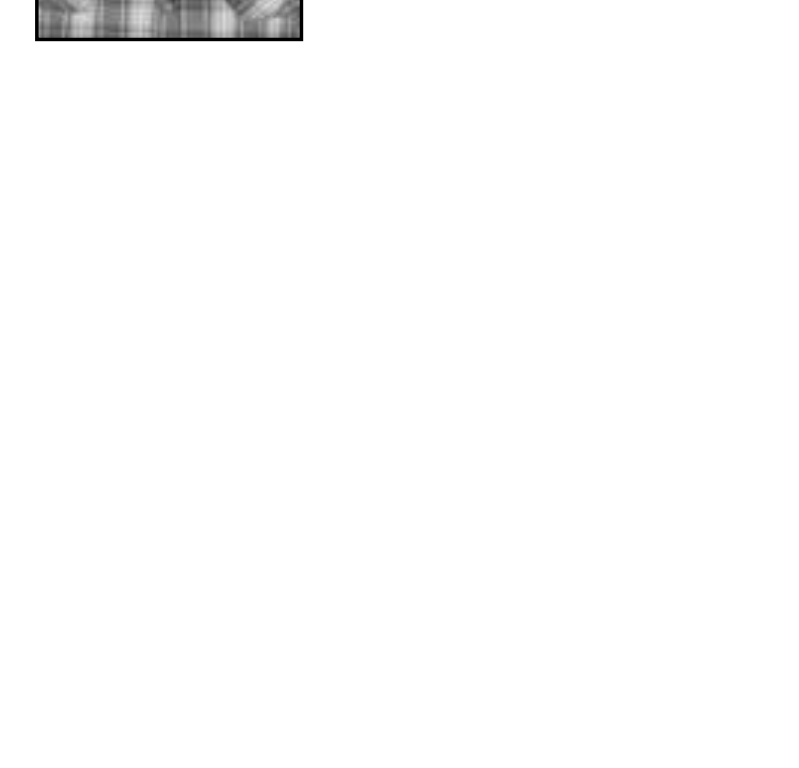

